\title{
Commentary on: Tissue "valve-over-valve" implantation in previous mechanical Bentall
}

\author{
Mohammed Hassan ${ }^{1}$ \\ ${ }^{1}$ Scott and White Memorial Hospital
}

July 21, 2020

\begin{abstract}
Mohammed Farouk Hassan, MD MBA ${ }^{1}$

${ }^{1}$ Department of Surgery, Baylor Scott \& White, Temple TX

Word Count: 472

Key Words: valve repair/replacement

Corresponding Author:

Mohammed F. Hassan, MD FACS MBA

Associate Professor, Texas A\&M University HSC COM

Baylor Scott \& White Health

Division of Cardiothoracic Surgery

Department of Surgery

Temple, TX

mhassanct@gmail.com
\end{abstract}

The authors present a case report about elective replacement of a mechanical bental with a bioprosthetic valve. The authors describe a technique whereby the mechanical valve is 'broken' off it's mechanism and the new valve is sutured in the old cuff.

Commentary on: Tissue "valve-over-valve" implantation in previous mechanical Bentall

Cell 513-460-3499

Abstract:

The authors present a case report about elective replacement of a mechanical Bentall with a bioprosthetic valve. The authors describe a technique whereby the mechanical valve is 'broken' off its mechanism and the new valve is sutured in the old cuff.

In the recently submitted case report by Okiwelu and colleagues, the authors describe a challenging case of a young patient who underwent a Bentall procedure using a $31 \mathrm{~mm}$ ATS mechanical valve conduit (Medtronic, Minneapolis, MN) who then requested re-replacement with a bioprosthetic valve because of the impact of the audible clicks from the mechanical valve on his quality of life. The authors describe a technique whereby the mechanical valve was 'broken' off its mechanism and the new valve (29mm Edwards Inspiris Resilia, Edwards, Irvine, CA) was sutured to the sewing cushion of the carbon housing. 
This is an interesting approach but raises some questions and points to consider. The low profile of the supra-annular portion of the ATS made it possible for this approach. The other mechanical valves being used currently have a supra-annular component that would prohibit such an approach.

With the approach described, the carbon housing of the valve was left behind. This is a 45-year-old patient who will need two or more aortic interventions in the future if he remains with the bioprosthetic option. The 'housing' left behind will limit the use of future TAVRs especially if there is pannus formation within the housing.

Despite using precautions while fracturing the valve, it is difficult to have full control of the carbon components with blunt fracture and, as the authors mention, increase the risk of a neurologic complication. This patient did have a neurologic complication of mild left eyelid droop with meiosis despite having a negative MRI exam.

In addition, the technique of breaking off the leaflets, causes damage to the general lining of the remaining 'housing' and damages the carbon surface which could serve as a nidus for thrombus formation as described by the FDA's summary of safety and effectiveness data. This would argue that the patient in this case may benefit from long term anti-coagulation. ${ }^{1,2}$

Even though it involves a more involved surgery, was a full redo bio-Bentall considered given the age of the patient. Also, was a Ross procedure considered for this patient? Mazine and colleagues carried out a meta-analysis comparing the Ross procedure with mechanical aortic valve replacement and concluded that there was lower all-cause mortality compared with mechanical aortic valve replacement. ${ }^{3}$

References

1. Gott V.L., Alejo D.E., Cameron D.E. Mechanical heart valves: 50 years of evolution. Ann. Thorac. Surg. 2003;76:S2230-S2239.

2. FDA. ATS Open Pivot Bileaflet Heart Valve: Instructions for use. 2000. Maryland, USA.

3. Mazine A, Rocha RV, El-Hamamsy I, et al. Ross Procedure vs Mechanical Aortic Valve Replacement in Adults: A Systematic Review and Meta-analysis. JAMA Cardiol . 2018;3(10):978-987. doi:10.1001/jamacardio.2018.2946 